\title{
ON THE DEGREE OF APPROXIMATION OF FUNCTIONS BY FEJER MEANS
}

A. ZYGMUND

1. Continuous functions. It has been proved by S. Bernstein that if $f(x)$ is periodic and of the class $\operatorname{Lip} \alpha, 0<\alpha<1$, then the $(C, 1)$ means $\sigma_{n}(x)=\sigma_{n}(x ; f)$ of the Fourier series of $f$ satisfy the condition

$$
\sigma_{n}(x)-f(x)=O\left(n^{-\alpha}\right),
$$

uniformly in $x$. The result is false for $\alpha=1$. The place of (1.1) is then taken by

$$
\sigma_{n}(x)-f(x)=O(\log n / n),
$$

and, as simple examples show, the factor $\log n$ on the right cannot be removed (see, for example, A. Zygmund, Trigonometrical series, p. 62). It will be shown here that for power series the inequality (1.1) holds even for $\alpha=1$. More generally, we have the following theorem.

ThEOREM 1. Suppose that $f(x)$ is periodic, continuous, and that the Fourier series of $f$ is of power series type,

Then

$$
f(x) \sim \sum_{\nu=0}^{\infty} c_{\nu} e^{i \nu x}
$$

$$
\left|\sigma_{n-1}(x)-f(x)\right| \leqq A \omega(2 \pi / n),
$$

where $\omega(\delta)$ is the modulus of continuity of $f$ and $A$ is an absolute constant.

The proof is based on the following lemma.

LemMa. Suppose that

$$
g(x) \sim \sum_{-\infty}^{+\infty} \gamma_{\nu} e^{i \nu x}
$$

satisfies $|g(x+h)-g(x)| \leqq M|h|$. Then

$$
\left|\tilde{\sigma}_{n-1}(x)-\tilde{g}(x)\right| \leqq B M / n,
$$

where $\tilde{g}(x)$ is the function conjugate to $g(x)$ and $\tilde{\sigma}_{n}(x)$ are the $(C, 1)$ means of the series conjugate to (1.4).

For the proof of the lemma we note that

Received by the editors August 3, 1944. 


$$
\begin{aligned}
\tilde{g}(x) & =-\frac{1}{\pi} \int_{0}^{\pi}[g(x+t)-g(x-t)] \frac{1}{2} \cot \frac{1}{2} t d t \\
\tilde{\sigma}_{n-1}(x) & =-\frac{1}{\pi} \int_{0}^{\pi}[g(x+t)-g(x-t)] \\
& \cdot\left[\frac{1}{2} \cot \frac{1}{2} t-\frac{\sin n t}{n(2 \sin (t / 2))^{2}}\right] d t, \\
\tilde{g}(x)-\sigma_{n-1}(x) & =\frac{1}{\pi} \int_{0}^{\pi}[g(x+t)-g(x-t)] \frac{\sin n t}{n(2 \sin (t / 2))^{2}} d t \\
& =\frac{1}{\pi} \int_{0}^{\pi / n}+\frac{1}{\pi} \int_{\pi / n}^{\pi}=P_{n}+Q_{n},
\end{aligned}
$$

say. Since $|\sin n t| \leqq n \sin t \leqq n(2 \sin (t / 2))$ for $0 \leqq t \leqq \pi$,

$$
\left|P_{n}\right| \leqq \frac{1}{\pi} \int_{0}^{\pi / n} \frac{2 M t}{2 \sin (t / 2)} d t \leqq \frac{M}{\pi} \int_{0}^{\pi / n} \frac{t d t}{(2 / \pi) t / 2}=\frac{M \pi}{n}
$$

In order to estimate $Q_{n}$, we introduce the function

$$
\Lambda_{n}(t)=\frac{1}{\pi n} \int_{t}^{\pi} \frac{\sin n u}{(2 \sin (u / 2))^{2}} d u,
$$

and integrate by parts. By the second mean value theorem,

$$
\left|\Lambda_{n}(t)\right| \leqq \frac{2}{\pi n^{2}} \cdot \frac{1}{(2 \sin (t / 2))^{2}}<\frac{\pi}{2 n^{2} t^{2}} .
$$

The function $g$ is absolutely continuous and $\left|g^{\prime}(x)\right| \leqq M$ almost everywhere. Thus

$$
\begin{aligned}
\left|Q_{n}\right| \leqq & \frac{1}{\pi}\left|\left[(g(x+t)-g(x-t)) \Lambda_{n}(t)\right]_{\pi / n}^{\pi}\right| \\
& +\frac{1}{\pi}\left|\int_{\pi / n}^{\pi}\left[g^{\prime}(x+t)+g^{\prime}(x-t)\right] \Lambda_{n}(t) d t\right| \\
\leqq & \frac{1}{\pi} \cdot M \cdot \frac{2 \pi}{n} \cdot \frac{\pi}{2 n^{2}(\pi / n)^{2}}+\frac{2 M}{\pi} \int_{\pi / n}^{\pi}\left|\Lambda_{n}(t)\right| d t \\
< & \frac{M}{\pi n}+\frac{M}{n^{2}} \int_{\pi / n}^{\infty} \frac{d t}{t^{2}}=\frac{2}{\pi} \frac{M}{n} .
\end{aligned}
$$

This completes the proof of the lemma, with $B=\pi+2 / \pi$.

Suppose now that the Fourier series of $f$ is of power series type so 
that $\tilde{f}=-i f$. If $|f(x+h)-f(x)| \leqq M|h|$, then

$$
\left|\sigma_{n-1}(x)-f(x)\right|=\left|\tilde{\sigma}_{n-1}(x)-\tilde{f}(x)\right| \leqq B M / n .
$$

To complete the proof of Theorem 1, we introduce the function

$$
\begin{aligned}
f_{h}(x) & =\frac{1}{2 h} \int_{-h}^{h} f(x+t) d t=[F(x+h)-F(x-h)] / 2 h \\
& \sim \sum_{\nu=0}^{\infty} c_{\nu} e^{i \nu x}\left(\frac{\sin \nu h}{\nu h}\right),
\end{aligned}
$$

where $F(x)$ is a primitive of $f$. Hence $d f_{h} / d x$ exists, is continuous, and does not exceed $\omega(2 h) / 2 h \leqq \omega(h) / h$ in absolute value. Moreover, the Fourier series of $f_{h}$ is also of power series type. Now,

$$
\begin{aligned}
& \left|\sigma_{n-1}(x ; f)-f(x)\right| \\
& \quad \leqq\left|\sigma_{n-1}(x ; f)-\sigma_{n-1}\left(x ; f_{h}\right)\right|+\left|\sigma_{n-1}\left(x ; f_{h}\right)-f_{h}(x)\right|+\left|f_{h}(x)-f(x)\right| \\
& \quad=\alpha_{n}+\beta_{n}+\gamma_{n},
\end{aligned}
$$

say, and

$$
\begin{aligned}
& \gamma_{n}=\left|\frac{1}{2 h} \int_{-h}^{h}[f(x+t)-f(x)] d t\right| \leqq \omega(h), \\
& \beta_{n} \leqq B \frac{\omega(h)}{h} \cdot \frac{1}{n} \\
& \alpha_{n}=\left|\sigma_{n-1}\left(x ; f-f_{h}\right)\right| \leqq \max _{x}\left|f-f_{h}\right| \leqq \omega(h) .
\end{aligned}
$$

If we set $h=2 \pi / n$ and collect the results, we obtain (1.3) with $A=2+B / 2 \pi<4$.

2. Additional remarks. The foregoing proof of the lemma has certain disadvantages. First of all, it uses the result that a Lipschitz function is an indefinite integral, a fact which lies deeper than the assumptions of the lemma. Moreover, the argument does not work with the $L^{p}$ metric. These difficulties are avoided by the following somewhat longer variant of the proof of the lemma. For the sake of brevity we do not compute the absolute constants $C$ that occur in the proof.

Let $P_{n}$ and $Q_{n}$ have the same meaning as before, and let $\psi(x, t)$ $=f(x+t)-f(x-t)$. Hence

$$
\left|P_{n}\right| \leqq\left|\frac{1}{\pi} \int_{0}^{\pi / n} \psi(x, t) \frac{\sin n t}{n(2 \sin (t / 2))^{2}} d t\right| \leqq \int_{0}^{\pi / n}|\psi(x, t)| t^{-1} d t .
$$

Let $R_{n}(t)=1 / \pi n(2 \sin (t / 2))^{2}<1 / n t^{2}$. Then, for $n \geqq 1$, 


$$
\begin{aligned}
Q_{n}= & \int_{\pi / n}^{\pi} \psi(x, t) R_{n}(t) \sin n t d t \\
= & -\int_{0}^{\pi(n-1) / n} \psi(x, t+\pi / n) R_{n}(t+\pi / n) \sin n t d t \\
2 Q_{n}= & \int_{\pi / n}^{\pi(n-1) / n} \psi(x, t)\left[R_{n}(t)-R_{n}(t+\pi / n)\right] \sin n t d t \\
& +\int_{\pi / n}^{\pi(n-1) / n}[\psi(x, t)-\psi(x, t+\pi / n)] R_{n}(t+\pi / n) \sin n t d t \\
& -\int_{0}^{\pi / n} \psi(x, t+\pi / n) R_{n}(t+\pi / n) \sin n t d t \\
& +\int_{\pi(n-1) / n}^{\pi} \psi(x, t) R_{n}(t) \sin n t d t=I_{n}+J_{n}+K_{n}+L_{n},
\end{aligned}
$$

say.

By the mean-value theorem

$$
\left|R_{n}(t)-R_{n}(t+\pi / n)\right| \leqq C n^{-2} t^{-8},
$$

so that

$$
\left|I_{n}\right| \leqq C n^{-2} \int_{\pi / n}^{\pi-\pi / n}|\psi(x, t)| t^{-3} d t \leqq C n^{-2} \int_{\pi / n}^{\pi}|\psi(x, t)| t^{-3} d t .
$$

Since $R_{n}(t+\pi / n) \leqq 1 / n t^{2}$, and $\psi(x, t)-\psi(x, t+\pi / n)=\psi(x+t-\pi / 2 n, \pi / 2 n)$

we find

$$
-\psi(x-t-\pi / 2 n, \pi / 2 n)
$$

$$
\begin{aligned}
\left|J_{n}\right| \leqq & C n^{-1} \int_{\pi / n}^{\pi}|\psi(x+t-\pi / 2 n, \pi / 2 n)| t^{-2} d t \\
& +C n^{-1} \int_{\pi / n}^{\pi}|\psi(x-t-\pi / 2 n, \pi / 2 n)| t^{-2} d t .
\end{aligned}
$$

Moreover, since $R_{n}(t+\pi / n)<C n$ for $0 \leqq t \leqq \pi / n$,

Finally,

$$
\left|K_{n}\right| \leqq C n \int_{0}^{\pi / n}|\psi(x, t+\pi / n)| d t
$$

$$
\left|L_{n}\right| \leqq C n^{-1} \int_{\pi(n-1) / n}^{\pi}|\psi(x, t)| d t=C n^{-1} \int_{0}^{\pi / n}|\psi(x+\pi, t)| d t .
$$


By assumption, $|\psi(x, u)| \leqq M|u|$, uniformly in $x$. From this we immediately deduce that each of the terms $\left|P_{n}\right|,\left|I_{n}\right|,\left|J_{n}\right|,\left|K_{n}\right|$, $\left|L_{n}\right|$ is less than or equal to $C M / n$, and (1.5) is proved.

Suppose now that instead of the inequality $|g(x+h)-g(x)| \leqq M|h|$ we have

$$
\text { (2.1) } \begin{aligned}
M_{p}[g(x+h)-g(x)] & =\left\{\int_{0}^{2 \pi}|g(x+h)-g(x)| p d x\right\}^{1 / p} \\
& \leqq M|h|
\end{aligned}
$$

for some $p \geqq 1$. Then Minkowski's inequality for integrals shows that $M_{p}\left[P_{n}\right], M_{p}\left[I_{n}\right], M_{p}\left[J_{n}\right], M_{p}\left[K_{n}\right], M_{p}\left[L_{n}\right]$ are all less than or equal to $C M / n$. For example,

$$
\begin{aligned}
M_{p}\left[P_{n}\right] & \leqq \int_{0}^{\pi / n} M_{p}[\psi(x, t)] t^{-1} d t \leqq \int_{0}^{\pi / n} 2 M d t=2 M \pi / n \\
M_{p}\left[I_{n}\right] & \leqq C n^{-2} \int_{\pi / n}^{\pi} M_{p}[\psi(x, t)] t^{-3} d t \\
& \leqq 2 C M n^{-2} \int_{\pi / n}^{\infty} t^{-2} d t=C M / n,
\end{aligned}
$$

and similarly in other cases. Thus, under the hypothesis (2.1),

$$
M_{p}\left[\tilde{\sigma}_{n-1}(x)-\check{g}(x)\right] \leqq B M / n
$$

where $B$ is an absolute constant. By an argument similar to that by which Theorem 1 was deduced from the lemma, we obtain the following theorem.

THEOREM 2. Suppose that the Fourier series of $f(x)$ is of the power series type. Then

$$
M_{p}\left[\sigma_{n-1}(x)-f(x)\right] \leqq A \omega_{p}(2 \pi / n) \quad(p \geqq 1)
$$

where $\omega_{p}(\delta)=\sup _{|t| \leq \delta} M_{p}[f(x+t)-f(x)]$.

Theorems 1 and 2 hold for $(C, \alpha)$ means, whatever $\alpha>0$. The analogues for Abel means are immediate consequences of the CauchyRiemann equations.

MT. Holyoke College 